\title{
The Average Transmission Overhead for Broadcast Encryption
}

\author{
Sarang Aravamuthan ${ }^{1}$ and Sachin Lodha ${ }^{2}$ \\ 1 Advanced Technology Center, Hyderabad, India. \\ 2 Tata Research Development and Design Centre, Pune, India.* \\ E-mail: \{a.sarangarajan, sachin.lodha\}@tcs.com
}

\begin{abstract}
We consider broadcast encryption schemes wherein a center needs to broadcast a secret message to a privileged set of receivers. We prescribe a probability distribution $\mathcal{P}$ on the privileged set. In this setting, the transmission overhead can be viewed as a random variable over $\mathcal{P}$ and we define its expected value as the average transmission overhead (or ato).

Given $\mathcal{P}$, the Shannon's entropy function $H($.) provides a lower bound on the average number of bits required to identify every privileged set. This provides a natural lower bound for the ato in terms of $H(\mathcal{P})$. For session key distribution, we consider the subset cover framework and bound the ato in terms of the size of the cover. We further specialize our bound to accommodate storage constraints at receivers.

We consider two families of distributions for $\mathcal{P}$ that occur naturally in broadcast networks.

- Each receiver independently joins the privileged set with probability $p$.

- The privileged set is selected uniformly from a collection of subsets of receivers.

We evaluate the ato of some practical schemes such as the subset difference method, the LSD scheme and the Partition-and-Power scheme under these distributions. Our investigations lead us to conclude that each scheme is inherently tailored to perform optimally for specific distributions.
\end{abstract}

Keywords. Broadcast Encryption, Average Transmission Overhead, Subset Cover Framework, Shannon's Entropy.

\section{Introduction}

The average transmission overhead is an important statistical measurement of the effectiveness of communication systems. It finds applications in diverse fields such as video transmission over wireless networks [13], management of replicated data [14] and classification of access networks [16]. The overhead is determined by prescribing a probability distribution on some parameter of the system and using standard methods from probability theory (see [1] for a good introduction) to compute its value.

The setting in a broadcast encryption scheme consists of a broadcaster $\mathcal{B}$ and a collection of receivers $\mathcal{R}$. Periodically, $\mathcal{B}$ will want only some privileged subset $R \subseteq \mathcal{R}$ of receivers to avail the broadcast. To ensure this, $\mathcal{B}$ first broadcasts a message $\mathcal{M}$ that only the receivers in $R$ can decipher. The receivers are pre-configured with the right bits of information to achieve this. $\mathcal{M}$ includes a session key that is used for encrypting further broadcasts intended for $R$. The transmission overhead is the length of $\mathcal{M}$, that is, the amount of information (in bits) required to transfer the session key to $R$. Naturally, this overhead depends on $R$.

\footnotetext{
* Both ATC and TRDDC are research units of Tata Consultancy Services Limited.
} 
Suppose we impose a probability distribution $\mathcal{P}$ on the privileged set. The distribution is determined by the particular application the broadcaster intends to deploy. For instance, if the programs belong to the premium pay-per-view category, the privileged sets are more likely to be small and $\mathcal{P}$ should relect this fact.

We assume that $\mathcal{P}$ holds true for every broadcast and is not affected by past broadcast history. Let us denote the probability of a subset $R \subseteq \mathcal{R}$ being privileged as $\mathcal{P}(R)$. The transmission overhead may then be viewed as a random variable over $\mathcal{P}$ whose expected value we define as the average transmission overhead (or ato). In other words,

$$
\text { ato }=\sum_{R \subseteq \mathcal{R}} \mathcal{P}(R) \cdot \operatorname{to}(R) .
$$

where to $(R)$ is the transmission overhead for $R$.

We observe that the Shannon's entropy function [15]

$$
H(\mathcal{P})=-\sum_{R \subseteq \mathcal{R}} \mathcal{P}(R) \log \mathcal{P}(R)^{3}
$$

provides a lower bound on the average number of bits required to identify every privileged set. Since the broadcasts are independent, any broadcast encryption technique must provide for enough communication to allow identification of the privileged set. This observation translates into the following fact.

\section{Fact 1.}

$$
\text { ato } \geq H(\mathcal{P})
$$

\subsection{Related work}

To the best of our knowledge, the concept of average transmission overhead has never been studied before in the broadcast encryption context. Broadcast encryption itself is a well-studied topic though; see for instance [7,3,8] for an introduction. In [3], a broadcast scheme is proposed using polynomial interpolation and related vector formulation methods. Fiat and Naor [8] consider $k$ resilient broadcast schemes where coalitions of $k$ users not in the privileged set cannot recover the session key. Blundo et al study the trade-off between communication and storage in an information theoretic setting for unconditionally secure broadcast encryption schemes $[4,5]$. Luby and Staddon [11] use a combinatorial model to study this trade-off.

Subset cover schemes were introduced in [12]. The authors describe a subset difference method to cover the set of non-revoked users by a collection of disjoint subsets. Each non-revoked user uses the key corresponding to his subset to recover the session key. If $r$ is the number of revoked users and $n$ the total number of users, their scheme has a transmission overhead of $2 r-1$ messages, each receiver stores $O\left(\log ^{2} n\right)$ keys and the processing time at a receiver is $O(\log n)$ operations. These bounds are improved by Halevy and Shamir [9] using a Layered Subset Difference (LSD) scheme. Specifically, they reduce the storage size to $O\left(\log ^{3 / 2} n\right)$ while the processing time remains the same. However, the transmission overhead increases to $4 r$ messages.

\footnotetext{
${ }^{3}$ In this paper, all logs are in base 2.
} 
In $[10,6]$, the authors develop new methods to reduce transmission overheads in broadcast encryption. The methods are based on the idea of assigning one key per each partition using one-way key chains after partitioning the users. One method adopts skipping chains on partitions containing up to $p$ revoked users and the other adopts cascade chains on partitions with layer structure. The scheme using the former reduces the transmission overhead down to $\frac{r}{p+1}$ messages asymptotically as $r$ grows, and the scheme using the latter keeps the transmission overhead very small when $r$ approaches 0 , where $r$ is the number of revoked users. Combining the two schemes, the authors propose a new broadcast encryption scheme with least transmission overhead. These schemes require a large storage at a receiver though, and this storage may grow exponentially in some cases.

The Partition-and-Power scheme ( $\mathrm{PaP})$ was introduced in [2] wherein each user stores $m$ keys and the subset cover is formed by partitioning the set of users into $n /(\log m+1)$ equally sized sets and taking the union of all subsets of each set. A lower bound for the maximum transmission overhead for a subset cover scheme was determined and the $\mathrm{PaP}$ scheme was shown to achieve within a constant factor of this bound.

\subsection{Our Contribution}

In this article, we assume that keys are distributed using the subset cover method. These offer several advantages over other key distribution methods. For instance, the receivers in this scheme are stateless, i.e. once configured, the keys don't have to be updated. This scheme is also fully resilient in the sense that the session key cannot be computed by any coalition of non-receivers. The keys may either be explicitly stored as in the complete subtree method [12] and partition-andpower $(\mathrm{PaP})$ scheme [2] or computed through pseudo-random generators as in the subset difference (SD) method [12] or the layered subset difference (LSD) method [9].

Our main result is a generic lower bound for the ato in terms of the storage at a receiver, the distribution $\mathcal{P}$ and the key length (Theorem 4). Using this bound as the benchmark, we evaluate different subset cover schemes (SD, LSD and PaP) under two families of distributions encountered in practice. Our findings show that each scheme is inherently tailored to perform optimally for specific distributions.

Outline of the paper: In the next section, we introduce subset cover schemes and derive the expression for the ato. We specialize this bound in terms of the storage at receivers when all subset keys are explicitly stored with the receiver. In Section 3, we consider some probability models for the privileged set that are natural and useful in practice, and compute the bounds for ato under these distributions. In Section 4, we consider some specific subset cover schemes (such as SD, LSD, $\mathrm{PaP}$ schemes) and estimate their ato for these distributions. We conclude with Section 5.

\section{The Subset Cover Framework}

We consider subset cover schemes introduced in [12]. The idea is to express the privileged set as a union of a collection of subsets from a cover and encrypt the session key with the subset key corresponding to each subset in the union. These encrypted values are broadcast. Each privileged user either stores the subset keys explicitly or is able to compute them using some pre-stored information. The subset key is then used to recover the session key from the encrypted message. 
Let $\mathcal{R}=\left\{r_{1}, \ldots, r_{n}\right\}$ be a set of $n$ receivers capable of receiving transmissions from a broadcaster $\mathcal{B}$. Let $\mathcal{S}, \mathcal{X} \subseteq 2^{\mathcal{R}}$ be collections of subsets of $\mathcal{R}$ defined in the following manner. $\mathcal{S}$ is the collection of privileged sets. In the most general case, $\mathcal{S}$ would comprise of all subsets of $\mathcal{R}$. $\mathcal{X}$ is defined to be a cover for $\mathcal{S}$, i.e. every element in $\mathcal{S}$ can be expressed as a union of some elements from $\mathcal{X}$. Formally,

$$
\forall R \in \mathcal{S}, \exists X_{R} \subseteq \mathcal{X} \text { such that } \bigcup_{X \in X_{R}} X=R
$$

Let

$$
f_{\mathcal{X}}(R)=\min _{\substack{X_{R} \subseteq \mathcal{X}, \cup_{X \in X_{R}} X=R}}\left|X_{R}\right|
$$

For each $X \in \mathcal{X}$, there exists a (unique) subset key $K_{X}$ that is known only to the receivers in $X$. To distribute a session key $K$ to a set of receivers, say $R \in \mathcal{S}, \mathcal{B}$ first finds the smallest cover for $R$ by the elements from $\mathcal{X}$. Suppose it is $R=X_{1} \cup X_{2} \cup \cdots \cup X_{f}$ where $f=f_{\mathcal{X}}(R)$. Then $\mathcal{B}$ sends the following broadcast

$$
\left\langle\left[M_{X_{1}}, \ldots, M_{X_{f}}, E_{K_{X_{1}}}(K), \ldots, E_{K_{X_{f}}}(K)\right], E_{K}(M)\right\rangle
$$

where $E_{K_{X}}(K)$ is the encryption of $K$ under $K_{X}$ and $E_{K}(M)$ is the broadcast message $M$ encrypted under $K$. Here the message $M_{X_{i}}$ uniquely addresses $X_{i}$. Note that the quantity in square brackets in (1) is the transmission overhead.

Suppose all keys are $t$ bits in length (so that $|\mathcal{X}| \leq 2^{t}$ ) and let $M_{\mathcal{X}}(R)=\left|M_{X_{1}}\right|+\cdots+\left|M_{X_{f}}\right|$. Let to $\mathcal{X}(R)$ be the transmission overhead to target $R$ so that

$$
\text { to } \mathcal{X}(R)=M_{\mathcal{X}}(R)+f_{\mathcal{X}}(R) t
$$

To define the average transmission overhead, we assume a probability distribution $\mathcal{P}$ on the privileged sets. Assume that a privileged set $R \in \mathcal{S}$, occurs with probability $0<\mathcal{P}(R) \leq 1$ so that $\sum_{R \in \mathcal{S}} \mathcal{P}(R)=1$. The average transmission overhead is then the quantity

$$
\text { ato } \mathcal{X}=\sum_{R \in \mathcal{S}} \mathcal{P}(R) \cdot \operatorname{to} \mathcal{X}(R)
$$

We observe that ato $\mathcal{X}$ depends only on the cover and not on any specific privileged set. Since $R$ is identified by $M_{\mathcal{X}}(R)$, using (2) and Fact 1, we get

$$
\begin{aligned}
\text { ato } \mathcal{X} & =\sum_{R \in \mathcal{S}} \mathcal{P}(R) \cdot M_{\mathcal{X}}(R)+\sum_{R \in \mathcal{S}} \mathcal{P}(R) \cdot f_{\mathcal{X}}(R) t \\
& \geq H(\mathcal{P})+\text { ax } t
\end{aligned}
$$

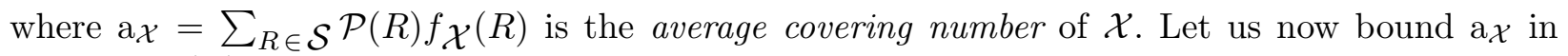
terms of $H(\mathcal{P})$.

\section{Claim 2.}

$$
\mathrm{a}_{\mathcal{X}} \log \frac{|\mathcal{X}|}{\mathrm{a} \mathcal{X}} \geq H(\mathcal{P})
$$


Proof. There are $|\mathcal{X}|$ different elements of $\mathcal{X}$. Let's suppose that they are represented by numbers 1 to $|\mathcal{X}|$. We denote by $N_{X}$ the number representing the set $X \in \mathcal{X}$.

Consider the case when $R \in \mathcal{S}$ is the privileged set whose minimum cover is $R=X_{1} \cup X_{2} \cup \ldots \cup X_{f}$ (where $f=f_{\mathcal{X}}(R)$ ), and $N_{X_{0}}=0<N_{X_{1}} \leq N_{X_{2}} \leq \cdots \leq N_{X_{f}} \leq|\mathcal{X}|$. Then, $\mathcal{B}$ sends $M_{X_{i}}=$ $N_{X_{i}}-N_{X_{i-1}}$ for $1 \leq i \leq f$. Note that

$$
N_{X_{i}}=\sum_{j=1}^{i} M_{X_{j}}
$$

so it is easy for receivers to reconstruct the numbers $N_{X_{i}}$ s and identify $X_{i}$ s. Here,

$$
\begin{aligned}
M_{\mathcal{X}}(R) & =\left|M_{X_{1}}\right|+\left|M_{X_{2}}\right|+\cdots+\left|M_{X_{f}}\right| \\
& =\log N_{X_{1}}+\log \left(N_{X_{2}}-N_{X_{1}}\right)+\cdots+\log \left(N_{X_{f}}-N_{X_{f-1}}\right) \\
& \leq f \log \frac{N_{X_{f}}}{f} \\
& \leq f_{\mathcal{X}}(R) \log |\mathcal{X}|-f_{\mathcal{X}}(R) \log f_{\mathcal{X}}(R) .
\end{aligned}
$$

Now,

$$
\begin{aligned}
H(\mathcal{P}) & \leq \sum_{R \in \mathcal{S}} \mathcal{P}(R) M_{\mathcal{X}}(R) \\
& \leq \sum_{R \in \mathcal{S}} \mathcal{P}(R) f_{\mathcal{X}}(R) \log |\mathcal{X}|-\sum_{R \in \mathcal{S}} \mathcal{P}(R) f_{\mathcal{X}}(R) \log f_{\mathcal{X}}(R) \\
& =\mathrm{a}_{\mathcal{X}} \log |\mathcal{X}|-\sum_{R \in \mathcal{S}} \mathcal{P}(R) f_{\mathcal{X}}(R) \log f_{\mathcal{X}}(R) \\
& \leq \mathrm{ax}_{\mathcal{X}} \log |\mathcal{X}|-\mathrm{a}_{\mathcal{X}} \log \mathrm{ax}_{\mathcal{X}}
\end{aligned}
$$

Note that we used the Jensen's inequality for convex function $\phi=x \log x$ in the final simplification. The Jensen's inequality says that $E[\phi(X)] \geq \phi(E[X])$ when $\phi$ is convex.

From Claim 2, we get the following bound on ax:

$$
\mathrm{a} \mathcal{X} \geq \frac{H(\mathcal{P})}{\log |\mathcal{X}|-\log \mathrm{a}_{\mathcal{X}}} \geq \frac{H(\mathcal{P})}{\log |\mathcal{X}|-\log H(\mathcal{P})+\log \log |\mathcal{X}|}
$$

Plugging this bound in equation (3), we get the following important lemma.

Lemma 3. Under a probability distribution $\mathcal{P}$ over the privileged sets $\mathcal{S} \subseteq 2^{\mathcal{R}}$, with a subset key of length $t$ bits, the average transmission overhead is bounded by

$$
\text { ato } \mathcal{X} \geq H(\mathcal{P})\left(1+\frac{t}{\log |\mathcal{X}|-\log H(\mathcal{P})+\log \log |\mathcal{X}|}\right)
$$

for broadcast encryption schemes that use subset cover method as outlined by (1). 
Suppose a receiver can store a maximum of $m$ subset keys and we consider schemes where the subset keys are stored explicitly in the receiver. The cover size then satisfies $|\mathcal{X}| \leq m n$ and (4) specializes to the following result.

Theorem 4. Under a probability distribution $\mathcal{P}$ over the privileged sets $\mathcal{S} \subseteq 2^{\mathcal{R}}$, with a storage bound of $m$ keys per receiver and a subset key of length $t$ bits, the average transmission overhead is bounded by

$$
\text { ato } \mathcal{X} \geq H(\mathcal{P})\left(1+\frac{t}{\log m n-\log H(\mathcal{P})+\log \log m n}\right)
$$

for broadcast encryption schemes that use subset cover method as outlined by (1) and explicit storage of subset keys at the receiver.

Theorem 4 bounds the average transmission overhead in terms of the parameters of the broadcast network, such as the number of receivers, key length, receiver storage and the distribution of the privileged set. Thus a practical scheme can determine its effectiveness by measuring how far it deviates from this bound.

\section{Some Special Probability Distributions}

We evaluate the lower bound expression in (5) for a couple of probability distributions that are natural and useful in practice.

\subsection{Independent and Identically Distributed Case}

Consider the case where each receiver, independently, joins the privileged set with probability $0 \leq p \leq 1$. Therefore, the probability of $R \subseteq \mathcal{R}$ being privileged is

$$
\mathcal{P}(R)=p^{|R|}(1-p)^{n-|R|} .
$$

For such a distribution $\mathcal{P}_{p}$ over $2^{\mathcal{R}}$, its Shannon entropy is

$$
H\left(\mathcal{P}_{p}\right)=n H_{2}(p)
$$

where $H_{2}(p)=-p \log p-(1-p) \log (1-p)$ is the standard binary entropy function. This follows from the fact that the entropy of the joint distribution of independent events is the sum of the individual entropies of each distribution.

Thus, equation (5) translates into

$$
\text { ato } \mathcal{X} \geq n\left(1+\frac{t}{\log m+\log \log m n}\right) H_{2}(p) .
$$

This expression is symmetric around $p=1 / 2$. It reaches its maximum value $n\left(1+\frac{t}{\log m+\log \log m n}\right)$ when $p=1 / 2$ which corresponds to the case where every subset of $\mathcal{R}$ is equally likely to be a privileged set. It is interesting to note that this bound is comparable with the lower bound of [2] for maximum transmission overhead in broadcast communication.

Note that equation (6) shows that the average transmission overhead is high for mid-range $p$. In fact, broadcast schemes addressing such distributions are hard to implement since all subsets of $\mathcal{R}$ are more or less equally likely to be privileged. 


\subsection{Equiprobable Case}

Next, we assume a uniform distribution over $\mathcal{S} \subseteq 2^{\mathcal{R}}$, i.e. all privileged sets occur with the same probability (of $1 /|\mathcal{S}|$ ). Let us label this probability distribution as $\mathcal{P} \mathcal{S}$. In this case, its Shannon entropy is

$$
\begin{aligned}
H\left(\mathcal{P}_{\mathcal{S}}\right) & =-\sum_{\substack{R \in \mathcal{S} \\
\mathcal{P}}} \mathcal{P}(R) \log \mathcal{P}(R)=\sum_{R \in \mathcal{S}} \frac{\log |\mathcal{S}|}{|\mathcal{S}|} \\
& =\log |\mathcal{S}| .
\end{aligned}
$$

Thus, equation (5) translates into

$$
\text { ato } \geq \log |\mathcal{S}| \cdot\left(1+\frac{t}{\log m n-\log \log |\mathcal{S}|+\log \log m n}\right) .
$$

When $\mathcal{S}=2^{\mathcal{R}}, \mathcal{P}_{\mathcal{S}}$ is the uniform distribution over all subsets of $\mathcal{R}$, and the expression in (7) reaches its maximum of $n\left(1+\frac{t}{\log m+\log \log m n}\right)$. The scenario of practical interest is the case where $\mathcal{S}=\{R \subseteq \mathcal{R}: l \leq|R| \leq u\}$, i.e., privileged sets are restricted to a size in the interval $[l, u]$. Then,

$$
|\mathcal{S}|=\left(\begin{array}{l}
n \\
l
\end{array}\right)+\cdots+\left(\begin{array}{l}
n \\
u
\end{array}\right) \geq \begin{cases}\left(\begin{array}{c}
n \\
n / 2
\end{array}\right) & \text { if } l \leq n / 2 \leq u \\
(u-l+1)\left(\begin{array}{c}
n \\
\frac{l+u}{2}
\end{array}\right) & \text { otherwise }\end{cases}
$$

This bounds $|\mathcal{S}|$ from below and hence the ato in equation (7).

\section{Analysis of Some Subset Cover Schemes}

We consider the SD, LSD and the PaP schemes and evaluate their ato values for distributions $\mathcal{P}_{p}$ and $\mathcal{P}_{\mathcal{S}}$ studied in Section 3.

\subsection{The Subset Difference method}

In the SD method [12], the storage per receiver is $m=O\left(\log ^{2} n\right)$ keys. The cover $\mathcal{X}$ is chosen in such a way that if $R$ is a privileged set of size $n-r$, then $f_{\mathcal{X}}(R) \approx 1.25 r$. The average covering number is then

$$
a_{\mathcal{X}} \approx \sum_{r=0}^{n}\left(\begin{array}{l}
n \\
r
\end{array}\right) 1.25 r p^{n-r}(1-p)^{r}=1.25 n(1-p) .
$$

Since identification of the privileged set in this scheme requires a negligible number of bits compared to key distribution, the ato value is

$$
\text { ato } \mathcal{X} \approx 1.25 n t(1-p)
$$

which decreases with $p$. Thus this scheme is effective when the privileged set is likely to be large. 


\subsection{The LSD method}

In the LSD method [9], the storage per receiver is $m=\log ^{3 / 2} n$ keys. The subset cover $\mathcal{X}$ satisfies $f_{\mathcal{X}}(R) \approx 2 r$ where $|R|=n-r$. Then $a_{\mathcal{X}}$ is calculated as

$$
a_{\mathcal{X}} \approx \sum_{r=0}^{n}\left(\begin{array}{l}
n \\
r
\end{array}\right) 2 r p^{n-r}(1-p)^{r}=2 n(1-p) .
$$

Thus this scheme is again effective when $p$ is large.

\subsection{The PaP Scheme}

Let $m$ be the number of subset keys that can be stored at a receiver. The PaP scheme [2] partitions $\mathcal{R}$ into groups of size $g:=\log m+1$. The subset cover $\mathcal{X}$ is the union of the power set of each of these groups. Thus, $|\mathcal{X}|=2^{g} n / g \approx 2 m n / \log m$. Each receiver stores all the keys corresponding to the subsets it belongs to in $\mathcal{X}$. To address a privileged set $R, R$ is expressed as a disjoint union of at most $f=n / g$ sets from $\mathcal{X}$. Thus for any distribution, the average cover number is always less than $n / g$.

4.3.1 Independent and Identically Distributed Case : Suppose $R \subseteq \mathcal{R}$ is privileged with distribution $\mathcal{P}_{p}$. If $f_{\mathcal{X}}(R)=j$, then $R$ contains at least one element from $j$ groups and none from the remaining groups. The average cover number is given by

$$
\begin{aligned}
a_{\mathcal{X}} & =\sum_{j=1}^{f} j\left(\begin{array}{l}
f \\
j
\end{array}\right)(1-p)^{g(f-j)}\left(\sum_{i=1}^{g}\left(\begin{array}{l}
g \\
i
\end{array}\right) p^{i}(1-p)^{g-i}\right)^{j} \\
& =f\left(1-(1-p)^{g}\right)=\frac{n}{g}\left(1-(1-p)^{g}\right)
\end{aligned}
$$

after routine simplifications. As one would expect, $a_{\mathcal{X}}$ increases with $p$. Now, the ato is given by

$$
\text { ato } \mathcal{X}=a_{\mathcal{X}}(\log |\mathcal{X}|+t)=\frac{n\left(1-(1-p)^{g}\right)\left(\log \frac{n}{g}+g+t\right)}{g} .
$$

Consider the ratio of ato $\mathcal{X}$ to the minimum ato. Using the lower bound provided by equation (6), we get

$$
\begin{aligned}
\frac{\text { ato } \mathcal{X}}{\min \text { ato }} & \leq \frac{n\left(1-(1-p)^{g}\right)\left(\log \frac{n}{g}+g+t\right)}{g} \cdot \frac{\log m+\log \log m n}{n H_{2}(p)(t+\log m+\log \log m n)} \\
& \leq \frac{2}{H_{2}(p)} \cdot\left(1+\frac{\log \log m n}{\log m}\right) .
\end{aligned}
$$

Note that we have used $g=\log 2 m \geq 2$, and $2^{t} \geq|\mathcal{X}| \geq n$ while deriving (8). There are two distinct terms in (8): the first term $\frac{2}{H_{2}(p)}$ underlines the probabilistic setup whereas the second term $\left(1+\frac{\log \log m n}{\log m}\right)$ represents the storage constraint. We now analyze this ratio for different values of $p$. 
$p=c / n$ (or $p=1-c / n$ ) where $c$ is a constant: In this case, the expression (8) is $\Theta(n)$. Thus, the performance of $\mathrm{PaP}$ scheme could be arbitrarily bad.

$p=1 / 2$ (or any constant): In this case, the expression (8) is $\Theta\left(1+\frac{\log \log m n}{\log m}\right)$. If $m$ is fixed, and $n$ is varied, then here again the performance of $\mathrm{PaP}$ scheme could be arbitrarily bad.

If $m n<2^{m}$ (which is a likely scenario in practice), then the expression (8) is $O(1)$. Thus, the ato of PaP scheme is within a constant factor of the optimal. For example, if $n=2^{20}, m=2^{10}$, and $p=1 / 2$, then the performance of $\mathrm{PaP}$ scheme is within a factor of 3 of the minimum on the ato measure.

4.3.2 Equi-Probable case : Since $\mathcal{S}$ can be any subcollection, the best we can do is bound the average cover number from above by $f=n / g$. Thus, the ato of $\mathrm{PaP}$ scheme is

$$
\text { ato } \mathcal{X} \leq 2 t f=\frac{2 t n}{\log 2 m} \text {. }
$$

Comparing with the lower bound given by (7), we observe that when $\log |\mathcal{S}| \approx n$, the ato is within a $\Theta\left(1+\frac{\log \log m n}{\log m}\right)$ factor of the minimum. When $|\mathcal{S}|$ is small, the PaP scheme is not effective.

\section{Conclusion}

The ato is a statistical measure of the efficacy of a transmission scheme and finds applications in different domains $[13,14,16]$. Thus studying this concept in the field of broadcast encryption is a natural extension. The ato can be used a yardstick to measure the effectiveness of a broadcast scheme and to compare different schemes under uniform conditions such as same storage at receivers, same key length and identical distributions.

In this article, we studied the subset cover framework and derived a lower bound for the ato under a given distribution on the privileged set. Our analysis used the entropy of the distribution to bound the number of bits required to identify any privileged set. We specialized the lower bound when the storage on a receiver was constrained. We considered two probability distributions and evaluated the ato of some subset cover schemes under these distributions. Our studies show that each scheme is naturally designed to give optimal performance for specific distributions.

Other distributions for the privileged set are possible. For instance, we have assumed that every receiver is privileged with the same probability $p$. A natural extension is to consider the scenario where different groups of receivers are privileged with different probabilities. The most general case would prescribe a probability for each privileged set. However, such distributions appear harder to analyze.

We emphasize that under a probabilistic setting, the ato is just one statistical measurement of the distribution. The overall efficiency of the broadcast scheme is determined by taking other statistical parameters into account such as the standard deviation, the minimum and maximum values of the transmission overhead etc.

\section{References}

1. N. Alon and J. Spencer, The Probabilistic Method, John Wiley \& Sons, Inc. 
2. S. Aravamuthan and S. Lodha. An Optimal Subset Cover for Broadcast Encryption. In S. Maitra et al. (Eds.), INDOCRYPT, Lecture Notes in Computer Science 3797, Springer-Verlag, 2005, pp. 221-231.

3. S. Berkovits. How to Broadcast a Secret. Advances in Cryptology-Eurocrypt'91, Lecture Notes in Computer Science 547, Springer 1991, pp. 536-541.

4. C. Blundo and A. Cresti. Space Requirements for Broadcast Encryption. Advances in Cryptology-Eurocrypt'94, Lecture Notes in Computer Science 950, Springer-Verlag, 1995, pp. 287-298.

5. C. Blundo, L. A. Frota Mattos and D. R. Stinson. Tradeoffs Between Communication and Storage in Unconditionally Secure Schemes for Broadcast Encryption and Interactive Key Distribution. Advances in CryptologyCRYPTO'96, Lecture Notes in Computer Science 1109, Springer-Verlag, 1996, pp. 387-400.

6. J. H. Cheon, N.-S. Jho, M.-H. Kim and E. S. Yoo. Skipping Cascade, and Combined Chain Schemes for Broadcast Encrypyion. Available at http://citeseer.ist.psu.edu/728634.html

7. G.H. Chiou and W.T. Chen. Secure Broadcasting Using the Secure Lock. IEEE Transactions on Software Engineering, Vol SE-15(8), August 1989, pp. 929-934.

8. A. Fiat and M. Naor. Broadcast Encryption. Advances in Cryptology-CRYPTO'93, Lecture Notes in Computer Science 773, Springer-Verlag, 1993, pp. 480-491.

9. D. Halevy and A. Shamir. The LSD Broadcast Encryption Scheme. Advances in Cryptology-CRYPTO'02, Lecture Notes in Computer Science 2442, Springer-Verlag, 2002, pp. 47-60.

10. N.-S. Jho, J. Y. Hwang, J. H. Cheon, M.-H. Kim, D. H. Lee and E. S. Yoo. One-way Chain Based Broadcast Encrypyion Schemes. Advances in Cryptology-EUROCRYPT 2005, Lecture Notes in Computer Science 3494 , Springer, 2005, pp. 559-574.

11. M. Luby and J. Staddon. Combinatorial Bounds for Broadcast Encryption. Advances in Cryptology-Eurocrypt'98, Lecture Notes in Computer Science 1403, Springer, 1998, pp. 512-526.

12. D. Naor, M. Naor and J. Lotspiech. Revocation and Tracing Schemes for Stateless Receivers. CRYPTO 2001, pp. 41-62.

13. D. Qiao and K. G. Shin. A Two-Step Adaptive Error Recovery Scheme for Video Transmission over Wireless Networks, Proceedings of IEEE Infocom, Tel Aviv, Israel, Mar. 2000; 1698-1704.

14. D. Saha, S. Rangarajan and S. Tripathi. An Analysis of the Average Message Overhead in Replica Control Protocols, IEEE Transactions on Parallel and Distributed Systems, Vol 7(10), 1996, pp. 1026-1034.

15. C. E. Shannon. A mathematical theory of communication. Bell Systems Technical Journal, Vol 27, 1948, pp. $379-423$ and $623-656$.

16. W. Wei, B. Wang, C. Zhang, J. Kurose and D. Towsley. Classification of Access Network Types: Ethernet, Wireless LAN, ADSL, Cable Modem or Dialup? Proceedings of IEEE Infocom (Infocom 2005), Miami, FL, USA, 2005.

17. T. Worsch. Lower and upper bounds for (sums of) binomial coefficients. Technical Report 31/94, Universität Karlsruhe, Fakultät für Informatik, 1994. Available online at http://www.ubka.uni-karlsruhe.de/cgibin/psgunzip/ira/1994/31/31.pdf 